\title{
AUTOEVALUACIÓN Y ACREDITACIÓN DEL PROGRAMA DE ODONTOLOGÍA DE LA USTA
}

María Claudia Latorre Gómez

Odontóloga, U. Santo Tomás, Magíster en Evaluación y Educación, U. Santo Tomás, Coordinadora de Autoevaluación de la Facultad de Odontología y del Área de

Autoevaluación y Regulación, CEPA, U. Santo Tomás, Seccional Bucaramanga.

Autor responsable de correspondencia: Dra. María Claudia Latorre G.

Correo electrónico: autoacad@ustabuca.edu.co

\begin{abstract}
RESUMEN
El presente artículo pretende registrar los apartes más significativos del proceso de autoevaluación con fines de acreditación, del programa de Odontología entre 2000 y 2003, con el propósito de motivar a la comunidad universitaria odontológica tomasina, a asumir, de manera eficaz, el plan de mejoramiento y las recomendaciones de la evaluación externa; crear los procesos y mecanismos necesarios para ello, y destinar los recursos humanos y económicos para garantizar su cumplimiento, a partir de las responsabilidades de cada uno de los actores, con el propósito de beneficiar a la comunidad con sus servicios. Su esencia se puede evidenciar en los documentos, registros de talleres, claustros, actas de comités, actas de Consejo de Facultad, informe de autoevaluación 2001, actualización 2002, informe de pares, sesiones de reflexión, entre otros, disponibles en los archivos de la Facultad y de la Seccional. [Latorre MC. Autoevaluación y acreditación del programa de Odontología de la Usta. Ustasalud Odontología 2004; 3: 7 - 12]
\end{abstract}

Palabras clave: Autoevaluación, Autorregulación, Acreditación

\section{SELF - EVALUATION WITH ACCREDITATION AIMS OF THE DENTISTRY PROGRAM FROM THE USTA}

\begin{abstract}
The present article intends to register the most significant facts from the process of self-evaluation with accreditation aims, of the Dentistry program between 2000 and 2003, in order to motivate the Dentist Community of Santo Tomás University, to assume, in effective way, the plan of improvement and the recommendations of the external evaluation; to create the necessary processes and mechanisms for it, and to destine the human and economic resources to guarantee its fulfillment, with the responsibilities of each one of the actors, in order to benefit the community with its services. Its essence can be demonstrated in documents, registries of workshops, meetings, acts of committees, acts of Faculty meetings, report of selfevaluatión 2001, update 2002, report of pairs, reflection sessions, among others, available in the archives of the Faculty in Bucaramanga.
\end{abstract}

Key words: Self-evaluation, Self-regulation, Accreditation.

\section{INTRODUCCIÓN}

La Facultad de Odontología de la Universidad Santo Tomás inició actividades académicas en 1979; desde entonces ha cumplido su compromiso social, en la región oriental del país, fruto de los esfuerzos continuos de directivos, docentes, estudiantes, empleados y egresados.

La autoevaluación y la regulación han sido fundamentales para la cualificación permanente de sus programas de pregrado y posgrado. En sus 25 años de existencia, la Facultad de Odontología de la USTA celebra el máximo reconocimiento, según las normas vigentes, de su programa de pregrado: Resolución de acreditación, 3164, de diciembre 11 de 2003, del Ministerio de Educación Nacional.
El auto-estudio llevado a cabo por el programa, sobre la base de los criterios, características, variables $e$ indicadores definidos por el Consejo Nacional de Acreditación, asumido por la facultad y la institución, con liderazgo y amplia participación de la comunidad universitaria, fue el punto de partida para la evaluación externa y el reconocimiento alcanzado.

Significativo registrar el ejercicio de metaevaluación, ${ }^{1}$ apoyado por la Vicerrectoría Académica, realizado a finales del 2001, por los pares colaboradores, doctores Sofía Jácome Liévano (Odontóloga, Magíster en Educación) y José Santiago Correa Uribe (Magíster en Educación de la Universidad de Antioquia) que permitió retroalimentar el proceso evaluativo, enriquecer el plan de mejoramien- 
to formulado y fortalecer la cultura de la autoevaluación y autorregulación. La Facultad de Odontología socializó y difundió aprendizajes y logros, que contribuyeron a impulsar los procesos de autoevaluación en la Seccional.

La dinámica de mejoramiento condujo a la actualización de la autoevaluación en el 2002; después del estudio y aprobación del informe y sus anexos, por las diferentes instancias, se envió el 19 de diciembre de 2002 al CNA la documentación de autoevaluación para el proceso de acreditación voluntaria.

Es motivo de orgullo para la institución haber tenido en la evaluación externa, de un distinguido equipo de pares académicos integrado por los doctores Ramsés Hakim Murad y Alberto Arango Botero. Sus aportes significativos para el mejoramiento de la calidad y su calidad humana y profesional convirtieron el desarrollo de la visita en un verdadero intercambio académico, que perdura en cada una de las acciones de la comunidad universitaria tomasina.

Cumplidas las etapas del proceso de acreditación: autoevaluación, evaluación externa y final, se obtuvo el reconocimiento de alta calidad, motivo de satisfacción para los directivos, estudiantes, docentes, egresados y empleados, quienes cumplieron a cabalidad con el lema: "Acreditación esfuerzo y compromiso de todos", en el marco de sus 25 años de funcionamiento. Este hecho reafirma el compromiso institucional con la cultura de calidad, a través de los procesos de autoevaluación y mejoramiento continuos; es un reto histórico para la Facultad.

\section{AUTOEVALUACIÓN}

El proceso siguió las guía de procedimiento del CNA, adaptándola a las particularidades del programa y de la institución. ${ }^{2}$ La fase de motivación, conceptualización y recopilación de la información estuvo a cargo del Comité de Autoevaluación de la Facultad, apoyado por las directivas y los departamentos académicos y administrativos. Los talleres de sensibilización, el trabajo de campo, encuestas y entrevistas, se desarrollaron con una amplia participación y sentido de pertenencia de los diferentes actores. La población participante en el proceso de autoevaluación estuvo conformada por la comunidad universitaria de la Facultad de Odontología: Estudian- tes, docentes, directivos, egresados y administrativos. También participaron, a través de algunos estudios, empleadores y usuarios de los servicios.

La ponderación, ${ }^{3}$ como ejercicio previo a la recolección de información, se desarrolló mediante talleres en 2000 y 2002, para incorporar las características propuestas por el modelo de ASCOFAME sobre la relación docencia-servicio. De esta manera se logró obtener la asignación del peso relativo de cada una de las características, de los factores del modelo aplicado y su correspondiente justificación, y tomar, como referentes, el Proyecto Educativo Institucional, la misión, los propósitos, objetivos y la visión institucional y de la Facultad, el Plan General de Desarrollo de la USTA y el estado de desarrollo nacional e internacional de la formación en Odontología.

El mencionado proceso incluyó la concertación de las características esenciales como referentes de altos niveles de calidad para el programa: Misión institucional y compromiso con la calidad, responsabilidad institucional para ofrecer las condiciones requeridas para culminar los estudios, calidad de los profesores y niveles de formación, atención a los estudiantes, núcleo de investigadores, desarrollo profesoral, estímulo a la docencia califica$\mathrm{da}$, formación integral, interacción académica, interdisciplinariedad, trabajos finales, actualización curricular, plan de aprendizaje para la docencia-servicio y su evaluación, servicios de bienestar, organización administrativa del programa, influencia sobre el entorno, desempeño de los egresados, planta fisica adecuada para la formación del profesional.

La ponderación realizada por el programa es muy cercana a la presentada por la comisión evaluadora, hecho que manifiesta la transparencia del proceso adelantado. Las características esenciales definidas por la Facultad fueron destacadas por la evaluación externa.

El análisis de la información recopilada en el proceso evaluativo permitió identificar aspectos positivos, que se busca mantener y fortalecer, y aspectos negativos del programa catalogados, como desafios, que hacen parte del plan de autorregulación formulado. Los juicios sobre cada uno de los factores se relacionan directamente con el cumplimiento de las características esenciales definidas; fueron validados en los talleres de docentes, estudiantes y egresados. A continuación se presentan los principales argumentos de la autoevaluación, que sustenta-

8 
ron el nivel del cumplimiento alcanzado en cada uno de los factores evaluados, según el modelo del CNA:

El factor I, Proyecto Institucional. Obtuvo un alto grado de cumplimento. Se destacó la claridad de la misión institucional y de la facultad y el nivel de compromiso de los actores frente a su desarrollo. Son relevantes: el sentido humanista, la formación integral, la fundamentación ética, crítica y creativa. El Proyecto Educativo Institucional de la USTA es reconocido por la comunidad universitaria y por los expertos externos e influye significativamente en la región. El desarrollo integral humano es el marco que integra el quehacer educativo de la institución. Orienta las acciones sobre la docencia, la investigación y la proyección social. El quehacer universitario tomista es de pertinencia social bajo los principios de utilidad y bien común.

El factor II, Estudiantes y Profesores. Registró un cumplimiento aceptable. La interpretación obtenida de la suficiente disponibilidad profesoral para la docencia junto con el nivel de formación requerida; la relación favorable entre el número de estudiantes y docentes, los adecuados recursos tecnológicos para las prácticas clínicas y preclínicas; el suministro de materiales, de calidad reconocida, los estatutos y reglamentos estudiantiles y profesorales; el programa de asesoría de estudiantes, el sistema de registro estudiantil, la interacción docente con las comunidades académicas, la participación democrática de docentes, estudiantes y egresados en el Consejo Superior, en el Consejo Académico General, en el Consejo Académico Seccional y en el Consejo de Facultad; los esfuerzos institucionales por el desarrollo profesoral.

El factor III, Procesos Académicos. Evidenció un alto grado de cumplimiento a partir de la formación integral de los estudiantes, la estructura y la actualización del currículo; la calidad de los trabajos realizados por los estudiantes, los recursos bibliográficos y las políticas de adquisiciones; la infraestructura informática y de la biblioteca, el plan de aprendizaje para la docencia servicio, las prácticas extramurales, la organización y dotación de las clínicas, reconocidas por expertos nacionales y latinoamericanos; las preclínicas, el reconocimiento de los egresados por la formación recibida, los mecanismos participativos para la evaluación periódica del programa, el sistema de información de los procesos clínicoodontológicos (SIPCO).

La evaluación del factor IV, Bienestar Institucional, presentó un alto grado de cumplimento dada la excelente organización de los servicios de bienestar de la Seccional, los programas en las áreas de promoción y desarrollo humano, cultura y deporte; el Centro de Pastoral, las áreas recreativas que disfruta la comunidad universitaria y sus egresados; el reconocimiento de su contribución al ambiente favorable para desarrollar una comunidad académica. Existe coherencia en los esfuerzos institucionales por compartir los conocimientos, las cualidades y los valores, que favorecen el desarrollo integral humano. La formación espiritual y el liderazgo social fortalecen los principios de la Universidad y validan su misión.

El factor V, Organización, Administración y Gestión. Registró un alto grado de cumplimiento. Esta apreciación se fundamentó en el cumplimento de los criterios de integridad, idoneidad, responsabilidad, equidad, y eficiencia; una adecuada organización institucional seguida por la de la facultad; la correspondencia con las necesidades y objetivos propios del programa, la estructura de la División de Ciencias de la Salud, el liderazgo de las directivas de la facultad, la capacidad de gestión de la decanatura, el sentido de pertenencia de la comunidad universitaria, las inversiones en la red interna, la interconexión de las sedes, el acceso a internet; los procesos de planeación, comunicación, seguimiento y control, las dependencias académicas y administrativas que apoyan el desarrollo de las unidades académicas, la eficiencia de los sistemas de información, el control y seguimiento de la bioseguridad en las clínicas, el SIPCO, los medios de comunicación y publicaciones periódicas de la institución y de la Facultad.

El factor VI, Egresados e Impacto sobre el medio, demostró un alto grado de cumplimiento bajo los principios de desarrollo integral humano, socialización y bien común, libertad y corresponsabilidad, autonomía y libre determinación comunitaria. El accionar de la Facultad en la región sigue las orientaciones del Proyecto Educativo Institucional, a través de las clínicas odontológicas de las sedes de Bucaramanga y Floridablanca, y las prácticas extramurales comunitarias y clínicas desarrolladas desde su fundación. Los indicadores para evaluar el impacto son la cobertura de la atención, la calidad en la prestación del servicio y la satisfacción del usuario externo e interno. La facultad realiza programas de extensión y educación continuada a través de diplomados, talleres y seminarios, dirigidos al sector salud, a los docentes, estudiantes, egresados y odontólogos, en general; fomenta el vínculo de identidad y pertenencia de sus 


\begin{tabular}{|c|c|c|c|c|c|}
\hline FACTOR & DESCRIPCIÓN & $\begin{array}{c}\text { PESO } \\
\text { RFL.ATIVo }\end{array}$ & $\begin{array}{l}\text { APROXIMACIÓN AL } \\
\text { PESO RELATIVO }\end{array}$ & CATECORIA & $\begin{array}{l}\text { CUMPLIMIENTO } \\
\text { DEL FACTOR }\end{array}$ \\
\hline 1 & Proyecto Institucional & 15 & 12 & B & 80 \\
\hline 2 & Estudiantes y Profesores & 18 & 13 & c & 72.2 \\
\hline 3 & Procesos Académicos & 17 & 13.5 & B & 79.5 \\
\hline 4 & Bienestar Institucional & 11 & 8.6 & B & 78 \\
\hline 5 & $\begin{array}{l}\text { Organización, administración } \\
\text { y gestión }\end{array}$ & 12 & 10.1 & B & 84 \\
\hline 6 & $\begin{array}{l}\text { Egresados e impacto } \\
\text { sobre el medio }\end{array}$ & 13 & 10.5 & B & 81 \\
\hline 7 & Recursos fisicos y financieros & 14 & 12.9 & A & 92 \\
\hline
\end{tabular}

Tabla 1. Resultado global de la autoevaluación 2002, del programa de Odontologia de la Universidad Santo Tomás, Seccional Bucaramanga

egresados a través de diversas estrategias de comunicación, educación continuada, servicios y apoyo a la Asociación de Odontólogos Tomasinos, A.D.O.T.

El factor VII, Recursos físicos y Financieros, fue de pleno cumplimiento. La Seccional Bucaramanga y la Facultad de Odontología disponen de una planta física adecuada y suficiente para el desarrollo de las funciones sustantivas y de bienestar. La Facultad de Odontología tiene un edificio de cuatro pisos $\left(1.440 \mathrm{~m}^{2}\right)$ para el funcionamiento de las clínicas odontológicas en el Campus de Floridablanca, y en la sede Bucaramanga un área de $332.3 \mathrm{~m}^{2}$. El área total académica y administrativa es de $2.704 .3 \mathrm{~m}^{2}$ representada en clínicas, preclínicas, laboratorios y oficinas.

La Seccional cuenta con auditorios de excelentes condiciones, en sus dos sedes y de un polideportivo dotado con todos los requerimientos para el bienestar de la comunidad universitaria. El programa dispone de recursos presupuestales de funcionamiento e inversión adecuados a su naturaleza y a sus características.

En la tabla 1 se registra el resultado cuantitativo y cualitativo de la autoevaluación del programa.

\section{EVALUACIÓN EXTERNA}

La visita para la evaluación externa del programa se realizó durante los días 19 al 21 de agosto de 2003. A continuación se incluyen apartes del juicio de calidad del programa tomado del informe de los pares, presenta- do por los doctores Ramsés Hakim Murad y Alberto Arango Botero al Consejo Nacional de Acreditación, organizados por factores: ${ }^{4}$

Proyecto institucional: "Vale la pena resaltar como fortaleza la formación integral a la luz del pensamiento humanista y cristiano de Santo Tomás de Aquino dentro del contexto ético, social y económico del país".

Estudiantes y profesores: "Fue muy positivo encontrar un alto grado de pertenencia hacia la institución tanto en los profesores como en la mayoría de los estudiantes y administrativos. Esta es una fortaleza muy importante del programa". "El alto grado de cumplimiento en las características esenciales reflejan la definición de los objetivos institucionales y del programa y el compromiso de la comunidad académica por alcanzar altos niveles de calidad."El número y la formación de los profesores es adecuado para una buena formación de los estudiantes".

Procesos académicos: "El alto grado de cumplimiento del factor refleja la responsabilidad, coherencia, pertinencia, idoneidad e integridad de la institución y del programa, en el desarrollo de su función docente, investigativa y de proyección social. La biblioteca es completa, la hemeroteca y las bases de datos son actualizadas, con salas de informática conectadas a Internet y al consorcio de Proquest con el Icfes. El alto grado de cumplimiento del factor como un todo demuestra que en los procesos académicos se da otra de las fortalezas del programa." "El programa a través de todas sus acciones, asegura una formación integral basada en los principios del cristianismo." 
Bienestar Institucional: "Se destaca el interés y calidad de las acciones que se llevan a cabo para el desarrollo del bienestar universitario de la comunidad, en los aspectos culturales, de salud, deportivos, de la convivencia y de la solidaridad. Los recursos y los espacios fisicos son un ejemplo institucional en la educación superior."

El juicio sobre la calidad del factor bienestar universitario estructurado por la facultad fue considerado bajo por parte de los pares teniendo en cuenta los servicios y las facilidades con que cuenta la institución. En la actualidad la comunidad universitaria de la facultad registra un mayor reconocimiento de los servicios ofrecidos por el Departamento de Bienestar Universitario. Es muy posible que en posteriores evaluaciones los estudiantes, docentes, administrativos y aun los egresados puedan calificar con mayor conocimiento estos esfuerzos institucionales.

Organización, administración y gestión:"La organización de la Institución y del programa es bueno. La gente conoce las instancias de la organización y de la administración. El trato por el personal directivo, administrativo y técnico es altamente positivo. Es notorio el interés que las personas muestran por sus funciones en el desarrollo del cargo."

Egresados e impacto sobre el medio: "Los egresados son importantes en la profesión odontológica del oriente colombiano." "Los egresados consideran tener una base sólida para el ejercicio profesional..."

Recursos fisicos y financieros: "El programa cuenta con una excelente planta física, las clínicas, preclínicas, laboratorios, aulas, oficinas, salas de profesores y, en general, todo lo relacionado con la infraestructura es de lo mejor en los programas de odontología." "La institución reinvierte sus excedentes económicos en su propio desarrollo y en el mejoramiento de sus recursos físicos, tecnológicos, educativos y científicos, en el bienestar humano de la comunidad universitaria y en el mejoramiento de la calidad en todos sus campos de acción."

El equipo de evaluadores presentó las siguientes recomendaciones para el mejoramiento de la calidad del programa que fueron aceptadas y agradecidas por la institución. Son parte del plan de mejoramiento formulado y en la actualidad registran avances dado el compromiso de la institución y de la facultad con su permanente cualificación:
"Estudiar cómo mejorar el promedio de permanencia de la carrera y cómo disminuir la deserción estudiantil; adoptar el sistema de créditos académicos teniendo en cuenta las alternativas de flexibilidad curricular; estudiar y analizar el sistema de evaluación de las clínicas, ampliar el rango salarial entre las diferentes categorías del profesorado; establecer en el reglamento profesoral estímulos a la docencia calificada y a la actividad investigativa del docente; preparar un plan de formación profesoral que cubra la formación en ciencias básicas y en investigación; ensayar todo tipo de estrategias para mejorar la participación y seguimiento de los egresados, así como el impacto sobre el medio."

\section{AUTORREGULACIÓN}

Ante los retos que se plantea a la educación superior y, en especial, a la formación de los profesionales de Odontología se requiere aprender continuamente e innovar en los procesos y estructuras académicas y administrativas. Por lo tanto, es la autorregulación, el conjunto de acciones planteadas a partir de los procesos y resultados de la autoevaluación, la base para incidir, de manera efectiva, en la cualificación del programa tanto en su interior como en su proyección e impacto social. ${ }^{5}$

La Universidad Santo Tomás Seccional Bucaramanga, a través de su facultad de Odontología, está comprometida con el plan de mejoramiento formulado a partir de la autoevaluación realizada, reforzado por la evaluación externa del programa y traducido en la actualidad en avances consolidados hacia la excelencia del programa en beneficio de sus estudiantes, egresados, docentes, administrativos, directivos, empleado y comunidad, en general.

La investigación es considerada esencial para la calidad del programa; por lo tanto, se presentan los siguientes comentarios, al respecto con el objeto de motivar la participación directa de la comunidad universitaria en su fortalecimiento.

En el plan de desarrollo de la Universidad Santo Tomás ${ }^{6}$ se encuentran formuladas las estrategias para fortalecer la cultura investigativa, se reconoce la necesidad de trabajar permanentemente para hacer visibles los grupos de investigación institucionales a través de la generación de productos nuevos de conocimiento, formación de investigadores, divulgación de los resultados, la apro- 
piación social y fortalecimiento de la interacción académica nacional e internacional.

En Colombia las facultades de Odontología se encuentran aunando esfuerzos por consolidar la cultura investigativa para aportar significativamente al ejercicio profesional. La Facultad de Odontología de la USTA, a través de la Asociación de Facultades de Odontología, ACFO, es partícipe en estos propósitos.

Los esfuerzos dirigidos por la División de Investigación de $\mathrm{ACFO}$, a través del plan estratégico evaluado durante el año 2003, retroalimentan el proyecto de fortalecimiento de la investigación formulado. La cooperación interinstitucional, la formación de docentes en maestrías y doctorados, el estímulo para investigadores son elementos fundamentales en este propósito.

La dinámica de regulación impulsada por la División de Educación de ACFO, a partir del proceso de construcción de los exámenes de estado de Odontología, ha enriquecido el compromiso de la facultad con el mejoramiento de sus prácticas evaluativas.

La evaluación del aprendizaje en las clínicas ha sido estudiada en la facultad. En la actualidad se avanza en la reestructuración de la evaluación de las clínicas integrales del adulto, partiendo de los avances de las clínicas integrales del niño; se registra la propuesta concertada de ajuste con los preparatorios como fundamento de evaluación por competencias.?

La adopción del sistema de créditos académicos es considerada por la Facultad una oportunidad para consolidar el interés por regular los procesos de aprendizaje, redireccionándolos hacia el fortalecimiento del aprendizaje autónomo; la incorporación de estrategias didácticas que promuevan el desarrollo de competencias, la planeación y seguimiento del trabajo independiente del estudiante y el uso de nuevas tecnologías, como apoyo a la docencia. El rediseño curricular por créditos académicos cumplió las diferentes etapas formuladas por la Seccional en el marco de su Política Curricular; ${ }^{8}$ se aplicará en el primer semestre del segundo período lectivo del 2004. Está previsto por la Facultad la evaluación y seguimiento de los resultados para su continuo perfeccionamiento.

La Asociación de Odontólogos Tomasinos, ADOT, se encuentra participando activamente en la cualificación del pregrado y posgrados; se espera ampliar la partici- pación de los egresados en la educación continuada y demás actividades programadas por la coordinación de egresados de la facultad, con el apoyo de la oficina de egresados de la Seccional. La Facultad espera una gran participación de sus egresados en las actividades programadas para la celebración de sus 25 años. El odontólogo tomasino continuará demostrando a la comunidad el resultado del proceso educativo sustentado en el equilibrio entre el saber, el hacer y el ser.

El liderazgo fuerte y explícito de la Facultad de Odontología en la cultura de autoevaluación y autorregulación, en la Seccional, ha generado cambios en los diferentes actores y en las instancias académicas y administrativas. El compromiso de directivos, docentes, estudiantes y egresados es trabajar por el crecimiento de las fortalezas institucionales y del programa halladas en el proceso, realizar el seguimiento constante de cada uno de los proyectos del plan de mejoramiento continuo, superar las debilidades y mantener el nivel de calidad alcanzado, y preparar el camino para competir por una acreditación internacional.

"Estamos en permanente rumbo hacia la excelencia y, sobre la base de lo ya construido con el esfuerzo de todos, podremos avanzar con mayor firmeza que ayer."

\section{BIBLIOGRAFÍA}

1. Facultad de Odontología, Universidad Santo Tomás Seccional Bucaramanga. Informe de autoevaluación, Documento central 2001.

2. Facultad de Odontología, Universidad Santo Tomás Seccional Bucaramanga. Informe de autoevaluación, Documento central Vol. I 2002.

3. Facultad de Odontología, Universidad Santo Tomás Seccional Bucaramanga. Informe de autoevaluación, Anexo B Ponderación Vol. 2002.

4. Hakim R, Arango A. Informe visita de pares académicos programa de Odontología de la Universidad Santo Tomás de Bucaramanga, Septiembre 19 de 2003.

5. Facultad de Odontología, Universidad Santo Tomás Seccional Bucaramanga. Informe de autoevaluación, Anexo F Regulación Vol. III 2002.

6. Universidad Santo Tomás, Plan general de desarrollo 2003.

7. Facultad de Odontología, Universidad Santo Tomás Seccional Bucaramanga. Reestructuración de los preparatorios. Julio 2004.

8. Facultad de Odontología, Universidad Santo Tomás Seccional Bucaramanga. Adopción de créditos académicos, Decreto 25666. Febrero 2004. 